\title{
Factors Determining Continuous Infusion Aerosol Delivery During Mechanical Ventilation
}

\author{
Michael McPeck, Sunya Ashraf, Ann D Cuccia, and Gerald C Smaldone
}

\begin{abstract}
BACKGROUND: Continuous nebulization of prostacyclins and albuterol by infusion pump during mechanical ventilation evolved as a popular off-label treatment for severe hypoxemic respiratory failure and asthma. Most institutions use a vibrating mesh nebulizer. A new breath-enhanced jet nebulizer is a potential alternative. This study was designed to compare these devices to better define factors influencing continuous infusion aerosol delivery. Device function, ventilator settings, and infusion pump flow were studied in vitro. METHODS: Using a bench model of adult mechanical ventilation, radiolabeled saline was infused at 6 flows $(1.5-12 \mathrm{~mL} / \mathrm{h})$ into test nebulizers; 4 examples of each were used in rotation to test device reproducibility. Four breathing patterns with duty cycles (percentage of inspiratory time) ranging from 0.13 to 0.34 were tested. The vibrating mesh nebulizer was installed on the "dry" side of the heated humidifier $\left(37^{\circ} \mathrm{C}\right)$. The breath-enhanced jet nebulizer, installed on the "wet" side, was powered by air at $3.5 \mathrm{~L} / \mathrm{min}$ and $50 \mathrm{psi}$. Infusion time was $1 \mathrm{~h}$. Inhaled mass of aerosol was collected on a filter at the airway opening. Inhaled mass was expressed as the percentage of the initial syringe radioactivity delivered per hour. Radioactivity deposited in the circuit was measured with a gamma camera. Data were analyzed with multiple linear regression. RESULTS: Variation in inhaled mass was significantly explained by pump flow and duty cycle $\left(\mathrm{R}^{2} \mathrm{0.92}\right)$ and not by nebulizer technology. Duty cycle effects were more apparent at higher pump flow. Vibrating mesh nebulizers failed to nebulize completely in $20 \%$ of the test runs. Mass balance indicated that vibrating mesh nebulizers deposited $15.3 \%$ in the humidifier versus $0.2 \%$ for breath-enhanced jet nebulizer. CONCLUSIONS: Aerosol delivery was determined by infusion pump flow and ventilator settings with comparable aerosol delivery between devices. The breathenhanced jet nebulizer was more reliable than the vibrating mesh nebulizer; $10-12 \mathrm{~mL} / \mathrm{h}$ was the maximum infusion flow for both nebulizer technologies. Key words: aerosols; nebulizers and vaporizers; inhalation administration; mechanical ventilators; humidifiers; drug delivery; bias flow; epoprostenol. [Respir Care 2021;66(4):573-581. (C) 2021 Daedalus Enterprises]
\end{abstract}

\section{Introduction}

Continuous infusion aerosol delivery during mechanical ventilation has evolved as a popular off-label treatment for severe respiratory failure with albuterol for airways (asthma) and with prostacyclins for parenchymal conditions

Mr McPeck, Dr Ashraf, and Dr Smaldone are affiliated with the Division of Pulmonary, Critical Care and Sleep Medicine, Department of Medicine, Stony Brook University Medical Center, Stony Brook, New York. Ms Cuccia is affiliated with the Respiratory Care Program, School of Health Technology and Management, Stony Brook University, Stony Brook, New York.

Mr McPeck presented a version of this paper at AARC Congress 2019, held November 9-12, 2019, in New Orleans, Louisiana. (eg, pulmonary arterial hypertension and adult respiratory distress syndrome). Administration of aerosolized prostacyclin during mechanical ventilation was originally conducted with a variety of pneumatic jet nebulizers using nebulizer flows (up to $8 \mathrm{~L} / \mathrm{min}$ ) with nebulizer filling by bolus dosing. ${ }^{1-7}$ In the past, dose control was managed by changing the concentration of prostacyclin solution infused into the nebulizer. ${ }^{7}$ In recent years, the Aerogen Solo

The study was supported in part by InspiRx (Somerset, NJ). The State University of New York at Stony Brook holds patents in the fields of nebulizer development and inhaled antibiotic delivery which have been licensed to InspiRx. Dr Smaldone and Ms Cuccia disclose relationships with InspiRx. Mr McPeck and Dr Ashraf have disclosed no conflicts of interest. 
vibrating mesh nebulizer (Aerogen, Galway, Ireland) has been marketed for use with mechanical ventilation and continuous infusion aerosol delivery by way of a "drop-bydrop (volumetric)" technique in which the drug solution concentration remains constant and inhaled delivery is adjusted by changing the infusion pump flow. ${ }^{8}$

While the vibrating mesh nebulizer appears to have largely displaced the use of jet nebulizers for continuous infusion aerosol delivery, only a single bench study has been published characterizing its delivery of inhaled aerosolized prostacyclin during mechanical ventilation in an adult lung model. ${ }^{9}$

Recently, the i-AIRE breath-enhanced jet nebulizer (InspiRx, Somerset, New Jersey) has been developed and tested for adult mechanical ventilation. ${ }^{10-11}$ Breath-enhanced jet nebulizer technology operates at a lower jet flow than typical jet nebulizers and has been reported to be more efficient than conventional jet devices. ${ }^{11}$ The breath-enhanced jet nebulizer has been investigated while positioned in the ventilator circuit on the wet (ie, outlet) port of the heated humidifier, where it has been determined to be less sensitive to humidification effects. ${ }^{10-11}$ The purpose of this study was to better define the factors that influence continuous infusion aerosol delivery during mechanical ventilation by comparing aerosol delivery by these 2 nebulizer technologies at different infusion pump flows. Nebulizer reproducibility and reliability over a range of ventilator settings were also measured.

\section{Methods}

\section{Experimental Setup}

This study was performed at the Pulmonary Mechanics and Aerosol Research Laboratory, Division of Pulmonary, Critical Care and Sleep Medicine, Department of Medicine, Stony Brook University Medical Center, Stony Brook, New York. The experimental setup is diagrammed in Figure 1. An Evita Infinity V500 critical care ventilator (Dräger, Telford, Pennsylvania) was used to ventilate a pair of 1-L neoprene test lungs with the 4 adult breathing patterns listed in Table 1 . The breathing patterns were selected to provide a range of duty cycles (ie, inspiratory time percentage, determined as inspiratory time divided by total cycle time). Bias flow was maintained at $2 \mathrm{~L} / \mathrm{min}$ for all test runs. Humidification was provided by a heated humidifier and dual-limb heated-wire ventilator circuit (MR-850, Fisher \& Paykel Healthcare, Ltd., Auckland, New Zealand, or Hudson RCI Conchatherm

Correspondence: Michael McPeck RRT FAARC, Division of Pulmonary, Critical Care and Sleep Medicine, 100 Nicolls Rd, T17-040 Health Sciences Center, Stony Brook, NY 11794-3869. E-mail: michael.mcpeck@stonybrookmedicine.edu.

DOI: $10.4187 /$ respcare.07715

\section{QUICK LOOK}

\section{Current knowledge}

Continuous infusion aerosol delivery is an off-label method that uses an infusion pump and a vibrating mesh nebulizer system in a "drop-by-drop" technique during mechanical ventilation. There are limited data available reporting delivered drug (ie, inhaled mass) for different nebulizer technologies in a humidified ventilator circuit.

\section{What this paper contributes to our knowledge}

We measured the influence of nebulizer technology, infusion pump flow, and ventilator settings on aerosol drug delivery. Drug delivery was a function of pump flow and ventilator duty cycle, but not nebulizer type. A new breath-enhanced jet nebulizer prototype was equivalent to vibrating mesh nebulization over the infusion pump flow range of $1.5-12 \mathrm{~mL} / \mathrm{h}$.

Neptune, Teleflex, Research Triangle Park, North Carolina) to provide fully saturated gas at $37 \pm 1^{\circ} \mathrm{C}$ for all experiments. The humidifier was operated in its invasive mode with default settings, and the temperature display was allowed to reach $37^{\circ} \mathrm{C}$ before each experiment commenced.

Four vibrating mesh nebulizers and 4 breath-enhanced jet nebulizers were used in rotation for all experiments. The experimental setup diagrammed in Figure 1 shows the breath-enhanced jet nebulizer in the circuit located at the outlet or wet side of the humidifier. For breath-enhanced jet nebulizer setup, the proximal temperature sensor of the heated wire ventilator circuit, which provides humidifier outlet temperature feedback to the humidifier controller, was relocated from the standard location on the proximal cuff of the inspiratory circuit limb to the point where the 2way selector valve was placed on the humidifier outlet, so that it remained exposed directly to humidifier outlet gas temperature and was not cooled by being downstream of the nebulizer. At the start of each test run, the 2-way selector valve was turned to direct ventilator gas flow through the breath-enhanced jet nebulizer, which was operated continuously at $3.5 \mathrm{~L} / \mathrm{min}$ using air at $50 \mathrm{psi}$.

The inset diagram in Figure 1 depicts the experimental setup for the vibrating mesh nebulizer, which was placed on the dry side of the humidifier as recommended by manufacturer's instructional literature. ${ }^{8}$ The vibrating mesh nebulizer was positioned in the circuit using the Aerogen adult T-piece, confirmed to be correctly connected and oriented according to manufacturer's instructions, and operated via an Aerogen Pro-X controller in its continuous mode. The Aerogen Pro-X controller and cable were checked for proper operation prior to each series of experiments. The 


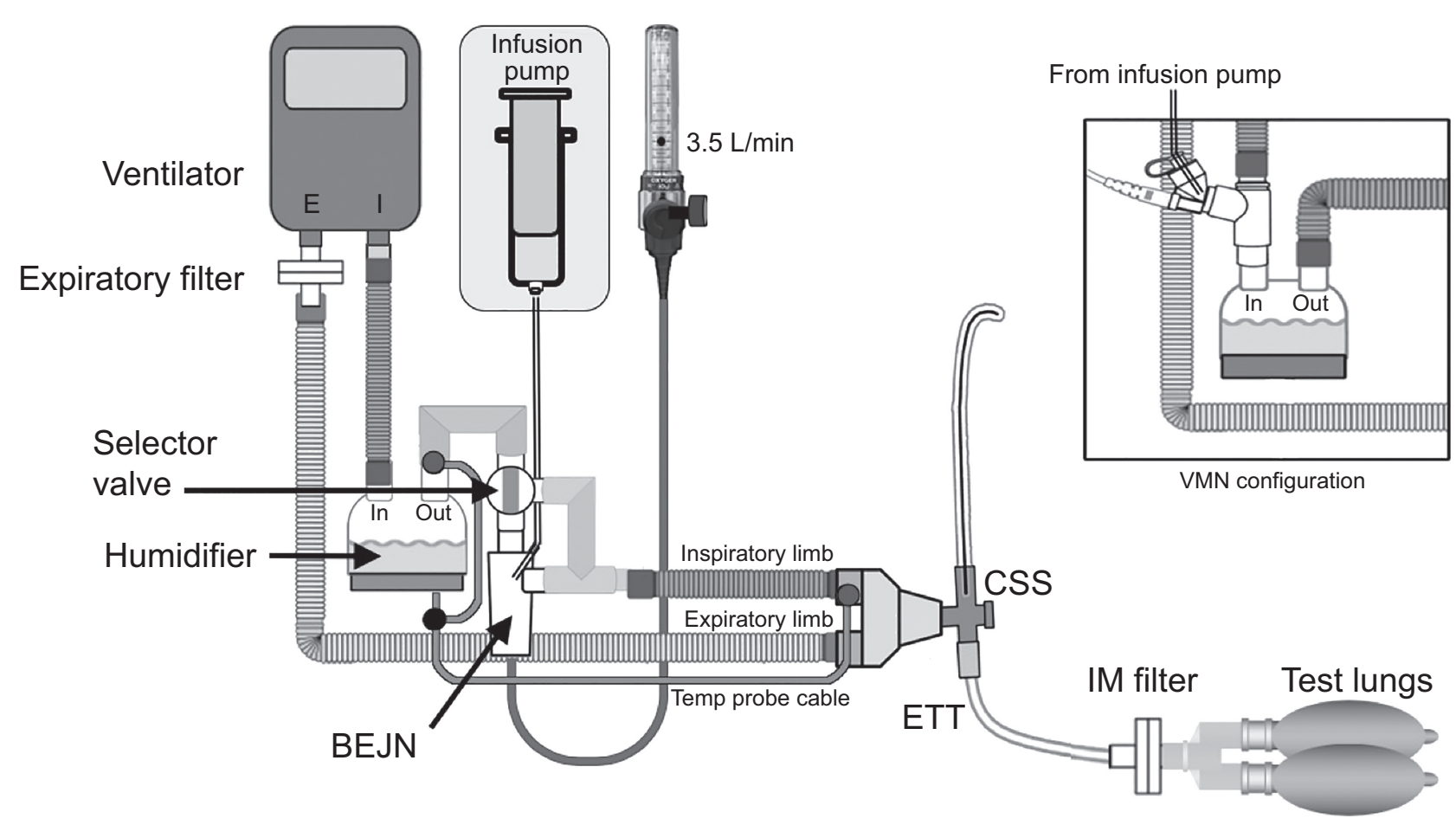

Fig. 1. Schematic diagram of experimental test setup depicting mechanical ventilator circuit and typical components to demonstrate mounting position and configuration of the breath-enhanced jet nebulizer on the wet side of the humidifier and the vibrating mesh nebulizer on the dry side of the humidifier (inset diagram). The breath-enhanced jet nebulizer is operated with $3.5 \mathrm{~L} / \mathrm{min}$ (at 50 psi) of medical air, oxygen, or blended gas. The selector valve allows the breath-enhanced jet nebulizer to be bypassed when not in use. The infusion pump infuses solution into each nebulizer type through its delivery tubing. The inhaled mass (IM) collection filter is shown distal to the closed system suction (CSS) device and endotracheal tube (ETT) and immediately proximal to the test lungs. The cascade impactor (not shown) was inserted via a T-connector into the circuit between the distal tip of the ETT and the IM filter for aerosol particle size determination.

Table 1. Ventilator Settings With Resulting Duty Cycles

\begin{tabular}{|c|c|c|c|c|c|c|c|}
\hline Ventilator Mode & $\begin{array}{c}\text { Breathing Frequency, } \\
\text { breaths/min }\end{array}$ & $\begin{array}{c}\text { Tidal Volume, } \\
\text { mL }\end{array}$ & $\begin{array}{l}\text { Minute Volume, } \\
\text { L/min }\end{array}$ & $\begin{array}{l}\text { Inspiratory Flow, } \\
\text { L/min }\end{array}$ & $\begin{array}{l}\text { Total Cycle } \\
\text { Time, s* }\end{array}$ & $\begin{array}{l}\text { Inspiratory } \\
\text { Time, s }\end{array}$ & $\begin{array}{l}\text { Duty } \\
\text { Cycle }\end{array}$ \\
\hline Volume control & 15 & 460 & 6.9 & 55 & 4.00 & 0.50 & 0.13 \\
\hline Volume control & 18 & 500 & 9.0 & 43 & 3.33 & 0.70 & 0.21 \\
\hline Pressure control & 15 & 494 & 7.4 & 31 & 4.00 & 0.95 & 0.24 \\
\hline Volume control & 20 & 650 & 13.0 & 39 & 3.00 & 1.01 & 0.34 \\
\hline
\end{tabular}

humidifier temperature sensor was inserted into its designated port on the proximal cuff of the inspiratory limb of the ventilator circuit attached to the humidifier outlet. All other aspects of the circuit were the same as for the breathenhanced jet nebulizer. The Aerogen controller was turned on in the continuous mode at the start of each test run. Run time was measured with a stopwatch.

To duplicate typical hospital ventilator circuits, the patient Y-piece was attached to a Ballard closed-system suction device (Avanos Medical, Alpharetta, Georgia), a 7.5-mm inner diameter endotracheal tube (Rusch, Teleflex
Medical, Morrisville, North Carolina), an inhaled mass collection filter (Pari, Starnberg, Germany) placed at the distal tip of the endotracheal tube to capture the aerosol particles that would be inhaled by a patient under similar conditions and, finally, to two 1-L neoprene test lungs connected in parallel via a Y-adapter to complete the inspiratory limb of the ventilator circuit. The expiratory limb of the circuit was connected to a second aerosol collection filter immediately prior to connection to the ventilator's exhalation channel. This filter was used to measure expiratory phase losses during aerosol delivery. 
Normal saline radiolabeled with $2-6 \mathrm{mCi}$ of technetium$99 \mathrm{~m}$ pertechnetate $\left({ }^{99 \mathrm{~m}} \mathrm{Tc}\right)$ was drawn into a $60-\mathrm{mL}$ infusion pump syringe (Aerogen P/N 06-AG-AS3085) to serve as a surrogate for water-soluble drugs. As an aqueous solution, radiolabeled saline behaves in nebulizers similarly to radiolabeled albuterol ${ }^{12}$ and epoprostenol. ${ }^{13}$ At the start of an experimental run, the nebulizer was dry, empty, and free of radioactivity. Priming was limited to completely filling only the infusion set delivery tubing and not permitting any solution to enter the nebulizer itself until the timed experimental test run was started.

Prior to each experiment, the radioactivity in the infusion pump syringe was measured with a gamma camera (Maxi Camera 400, General Electric, Horsholm, Denmark; Power Computing, Model 604/150/D, Austin, Texas; Nuclear MAC OS 4.2.2, Scientific Imaging, Thousand Oaks, California) to establish its initial charge. The actual time $\left(t_{0}\right)$ of the initial charge determination was recorded so that subsequent measurements could be decay-corrected for the time elapsed since $t_{0}$.

\section{Infusion Protocol}

Contemporary drug delivery for inhaled prostacyclin, for example, has been managed with a vibrating mesh nebulizer and a programmable infusion pump system in which the drug concentration $(\mu \mathrm{g} / \mathrm{mL})$ in the infusion syringe, the patient's ideal body weight $(\mathrm{kg})$ and the desired administration rate $(\mu \mathrm{g} / \mathrm{kg} / \mathrm{min})$ have been entered by the clinician. Based on those inputs, the infusion pump module calculates and delivers the appropriate flow of solution $(\mathrm{mL} / \mathrm{h})$ into the nebulizer to achieve the set drug delivery rate. Because the minimum output rate for all vibrating mesh nebulizers is $0.2 \mathrm{~mL} / \mathrm{min}$ (ie, $12 \mathrm{~mL} / \mathrm{h}$ ), and the maximum output rate is not specified, the manufacturer recommends a maximum input rate (to the nebulizer from the pump) of $12 \mathrm{~mL} / \mathrm{h} .{ }^{8}$ Consequently, our infusion protocol specified a range of infusion pump flow settings $(1.5,3.0,5.0,7.5,10$, and $12 \mathrm{~mL} / \mathrm{h})$, for each of the 4 breathing patterns and duty cycles listed in Table 1, thereby limiting the maximum infusion pump flow to $12 \mathrm{~mL} / \mathrm{h}$ for both nebulizer types. A programmable infusion syringe pump (Alaris Pump Module, Becton, Dickinson and Co., Franklin Lakes, New Jersey) was used to infuse radiolabeled saline into both nebulizer types. Each infusion experiment was run for $1 \mathrm{~h}$.

After commencing the experimental protocol, it became necessary to modify it to include measurement of excessive residual volume that remained in the vibrating mesh nebulizers after they had failed to start nebulization or failed to complete nebulization. These test runs were reported separately because the drop-by-drop technique expects all liquid infused into the nebulizer to be nebulized. To measure this effect, the retained solution was aspirated into a syringe through a small-bore needle to estimate its volume. This volume was defined as "retained volume" previously by Gowda and colleagues. ${ }^{14}$ Test runs that resulted in retained volume were repeated to obtain a complete data set for analysis of the vibrating mesh nebulizer.

After each test run, radioactivity captured on the inhaled mass filter was measured using the gamma camera and decay-corrected by the time elapsed since $t_{0}$. Aerosol delivered was expressed as inhaled mass delivered as a percentage of the initial charge in the syringe per hour.

Aerosol particle size distributions were determined by sampling the stream of radiolabeled saline particles emitted from the distal tip of the endotracheal tube with a 7-stage, low-flow cascade impactor (Marple 290 Series Cascade Impactor; ThermoFisher Scientific, Waltham, Massachusetts) at a vacuum flow of $2.5 \mathrm{~L} / \mathrm{min}$ for $30 \mathrm{~min}$ after the system had reached $37^{\circ} \mathrm{C}$ while operating at an infusion pump setting of $7.5 \mathrm{~mL} / \mathrm{h}$ and a ventilator duty cycle of 0.34 . Two nebulizers of each type were tested, and their results averaged. Radioactivity captured on each impactor stage was measured by a collimated ratemeter (Ludlum Measurements, Sweetwater, Texas) and plotted as log particle size $(\mu \mathrm{m})$ on the ordinate versus cumulative probability on the abscissa. Activity at the median (50\% probability) defined the mass median aerodynamic diameter (MMAD). ${ }^{15-16}$

A mass balance measurement was performed to quantify the distribution of radioactivity deposited throughout the ventilator circuit. Clean circuit components free of radioactivity were used. After $1 \mathrm{~h}$ of run time, each component of the ventilator circuit, including the humidifier chamber with its residual water, was separately placed on the gamma camera for measurement. In addition to the humidifier and its water together, the radioactivity of the residual water alone was determined by emptying it into a clean beaker for measurement on the gamma camera. Gamma camera images of all parts were saved digitally for measurement and inspection. Mass balance data are reported as a percentage of the radioactivity infused into the nebulizers over $1 \mathrm{~h}$.

\section{Analysis}

Aerosol delivery, expressed as inhaled mass (\% syringe charge/h) versus infusion pump flow $(\mathrm{mL} / \mathrm{h})$, was analyzed using multiple linear regression. The contributions of all potential variables, including infusion pump flow, nebulizer technology, and duty cycle, were assessed with this analysis. Data from the failed vibrating mesh nebulizer experiments were excluded from multiple regression analysis. Aerosol particle size distributions were plotted as log particle size versus probability to generate the cumulative probability graphs from which the MMAD was determined. Statistical analysis was conducted using GraphPad Prism 8.3 for Mac OS (GraphPad Software, San Diego, California). 

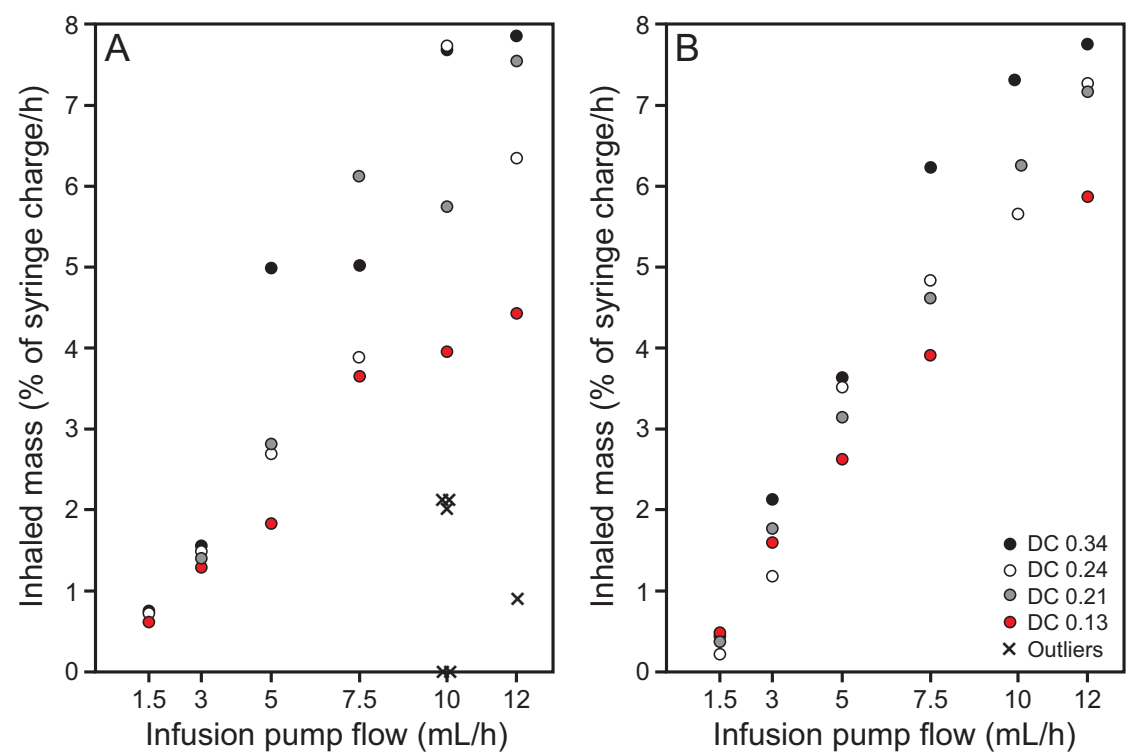

Fig. 2. Inhaled mass as a percent of syringe charge/h plotted against infusion pump flow for the vibrating mesh nebulizer (A) and the breathenhanced jet nebulizer (B). Data points represent 4 different ventilator duty cycles $(0.13,0.21,0.24$ and 0.34$)$ at each of 6 infusion pump flows $(1.5,3.0,5.0,7.5,10.0$ and $12.0 \mathrm{~mL} / \mathrm{h})$. There is a minimum of 4 data points plotted for each pump flow but occasional overlap partially or totally obscures visualization of some data points. $\mathrm{DC}=$ duty cycle.

Table 2. Aerogen Vibrating Mesh Nebulizer Conditions for Outlier Data Reported in Figure 2

\begin{tabular}{|c|c|c|c|c|c|c|}
\hline $\begin{array}{l}\text { Vibrating Mesh Nebulizer } \\
\text { Identification }\end{array}$ & $\begin{array}{l}\text { Infusion Pump Flow, } \\
\text { mL/h }\end{array}$ & $\begin{array}{l}\text { Duty } \\
\text { Cycle* }\end{array}$ & $\begin{array}{l}\text { Run Time, } \\
\text { min }\end{array}$ & $\begin{array}{l}\text { Inhaled } \\
\text { Mass }\end{array}$ & $\begin{array}{l}\text { Retained Volume, } \\
\mathrm{mL}\end{array}$ & Failure Mode \\
\hline 1 & 10 & 0.13 & 24 & 0 & 4.0 & Failed to start \\
\hline 3 & 10 & 0.13 & 12 & 0 & 2.0 & Failed to start \\
\hline 3 & 10 & 0.13 & 60 & 2.12 & 4.4 & Failed to keep up ${ }^{\S}$ \\
\hline 4 & 10 & 0.13 & 60 & 2.03 & 5.2 & Failed to keep up ${ }^{\S}$ \\
\hline 1 & 10 & 0.21 & 60 & 2.12 & 6.2 & Failed to keep up ${ }^{\S}$ \\
\hline 1 & 12 & 0.13 & 40 & 0.92 & 6.1 & $\begin{array}{l}\text { Stopped nebulizing at } 40 \mathrm{~min} \text {; would not } \\
\text { restart }^{\pi}\end{array}$ \\
\hline \multicolumn{7}{|c|}{$\begin{array}{l}\text { * Inspiratory time (s)/total cycle time (s). } \\
{ }^{+} \text {Percent of syringe charge. } \\
{ }^{2} \text { Test runs were discontinued prematurely when the vibrating mesh nebulizer failed to commence nebulization after repeated attempts within } 24 \text { and } 12 \text { min for nebulizer } 1 \text { and nebulizer } 3 \text {, respectively, } \\
\text { of being switched on. } \\
{ }^{\S} \text { Observed to develop excessive retained volume during test run resulting in measurable retained volume at completion of test run. } \\
\text { " Observed to develop excessive retained volume during test run and stopped nebulizing before the test run had completed. Measured retained volume approximated maximum fill volume of nebulizer res- } \\
\text { ervoir. Nebulizer would not restart. }\end{array}$} \\
\hline
\end{tabular}

\section{Results}

Data for all test runs plotted in Figure 2 are expressed as inhaled mass (\% of syringe charge/h) against infusion pump flow. Each data point represents radioactivity collected on an inhaled mass filter from 1 of the 4 nebulizers of each type tested, and for each of the 4 ventilator duty cycles. Figure 2 demonstrates that aerosol delivery (inhaled mass) increases linearly with increasing infusion pump flow, with increasing scatter of data with duty cycle at greater infusion pump flows. During the test runs for data collection, the vibrating mesh nebulizers did not function as intended in 20\% of total experiments. These outliers are marked with an X symbol in Figure 2 and summarized in Table 2 along with their failure mode. The breath-enhanced jet nebulizers did not experience any failures during the experiments.

With the vibrating mesh nebulizer outliers excluded from the calculation, multiple linear regression analysis revealed that variability in aerosol delivery was explained by infusion pump flow and duty cycle (Table 3 ). In the regression model, inhaled mass was set as the dependent variable, whereas nebulizer type, infusion pump flow, and duty cycle were defined 
Table 3. Multiple Linear Regression Analysis

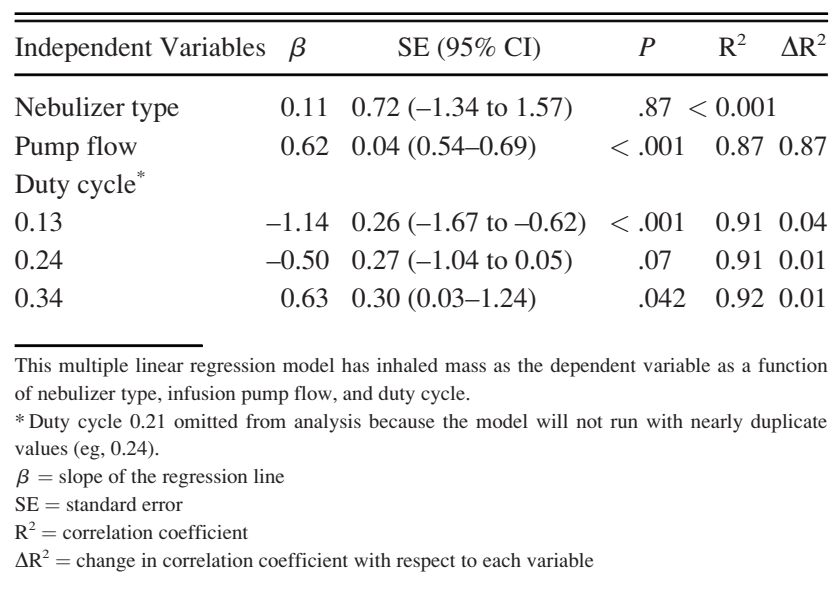

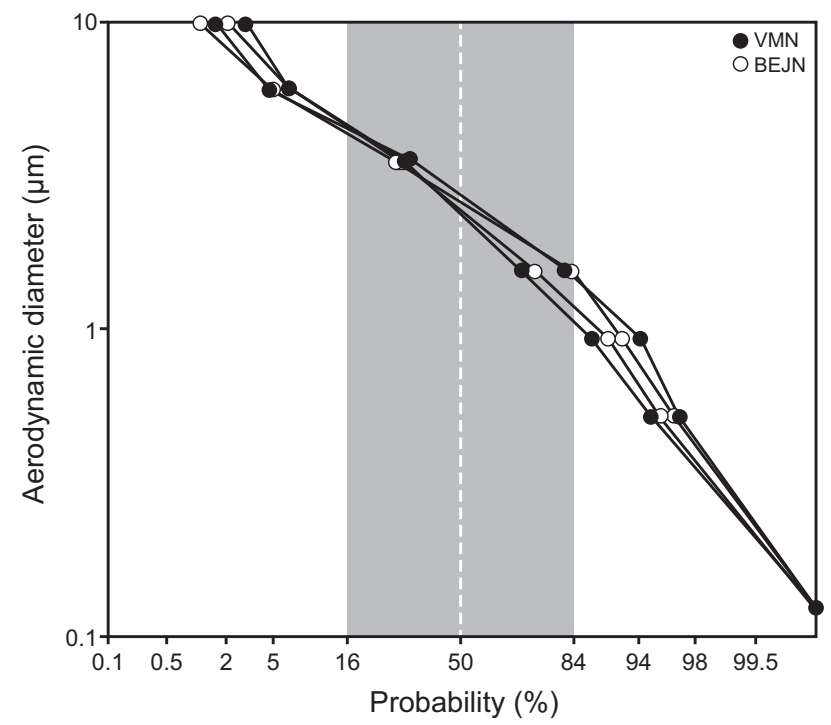

Fig. 3. Aerosol particle size distributions for breath-enhanced jet nebulizer (white circles) and vibrating mesh nebulizer (black circles) plotted as log particle size versus cumulative probability. Mean \pm SD mass median aerodynamic diameter at $50 \%$ probability was $2.54 \pm 0.23 \mu \mathrm{m}$ for the vibrating mesh nebulizer and $2.52 \pm 0.09$ $\mu \mathrm{m}$ for the breath-enhanced jet nebulizer.

as independent variables. This analysis revealed that there was no significant effect of nebulizer type on inhaled mass. Variability in aerosol delivery was closely related to infusion pump flow and inversely with duty cycle. The value of $\mathrm{R}^{2}$ (0.92) indicates that $92 \%$ of the variability of inhaled mass was accounted for by these variables; infusion pump flow, $87 \%$; and duty cycle an additional $5 \%$.

The aerosol particle size distributions determined with cascade impaction, and shown graphically in Figure 3, rendered a mean \pm SD MMAD of $2.54 \pm 0.23 \mu \mathrm{m}$ and $2.52 \pm$ $0.09 \mu \mathrm{m}$ for the vibrating mesh nebulizer and breathenhanced jet nebulizer, respectively.
Table 4. Mass Balance Determinations

\begin{tabular}{lcc}
\hline \hline & $\begin{array}{c}\text { Vibrating Mesh } \\
\text { Nebulizer }\end{array}$ & $\begin{array}{c}\text { Breath-Enhanced Jet } \\
\text { Nebulizer }\end{array}$ \\
\hline System components & & \\
Nebulizer residual & 2.8 & 20.7 \\
Nebulizer T-piece & 5.0 & 3.7 \\
Humidifier chamber & 15.3 & 0.2 \\
Inspiratory limb and & 20.1 & 15.1 \\
$\quad$ patient-Y & 1.3 & \\
Closed-system suction & & 1.1 \\
$\quad$ device & 1.2 & 3.3 \\
Endotracheal tube & 31.8 & 39.7 \\
Inhaled mass filter & 1.6 & 0.9 \\
Expiratory limb & 20.9 & 15.4 \\
Expiratory filter & 100 & 100 \\
Total mass balance &
\end{tabular}

Data are presented as percentage of radioactivity infused into nebulizer. Tests were conducted a an infusion pump flow of $10 \mathrm{~mL} / \mathrm{h}$ and ventilator duty cycle of 0.34 .

The mass balance measurements are shown in Table 4 . The data represent the distribution of radioactivity deposited on different components of the system and expressed as a percentage of the total radioactivity delivered to a representative sample of each nebulizer type during a 1-h test run. The humidifier retention of aerosol showed that the vibrating mesh nebulizers' dry-side placement deposited $15.3 \%$ in the humidifier compared to $0.2 \%$ for the breathenhanced jet nebulizers' wet-side placement. Of the $15.3 \%$ deposited in the humidifier, $80 \%$ of that was contained in the water while $20 \%$ was deposited on the inside of the humidifier chamber itself, predominantly on the inlet and outlet ports as determined by visual inspection of the humidifier scan image. Aerosol behavior in the rest of the circuit was comparable between both devices except for a higher residual in the breath-enhanced jet nebulizer.

\section{Discussion}

Our results indicate that, for both nebulizer technologies used during continuous infusion, the determining factors controlling aerosol drug delivery rate to the distal tip of the endotracheal tube are the infusion pump flow and the duty cycle of the ventilator. The 2 nebulizer technologies delivered comparable inhaled mass of radiolabeled saline across the range of infusion pump flows studied. This finding suggests that the 2 different nebulizer technologies can be used interchangeably within existing hospital-devised protocols for continuous infusion aerosol delivery.

The manufacturer specifies a minimum output rate for the vibrating mesh nebulizer of $0.2 \mathrm{~mL} / \mathrm{min}$, or $12 \mathrm{~mL} / \mathrm{h}^{8}$ and specifically advises against an infusion flow that exceeds the $12 \mathrm{~mL} / \mathrm{h}$ output rate of the device. No maximum output rate is specified by the manufacturer, although vibrating mesh 
nebulizers have been reported to function during continuous infusion aerosol delivery at output rates $>12 \mathrm{~mL} / \mathrm{h}$ (eg, 20.2 $\mathrm{mL} / \mathrm{h}$ when pretested to ascertain maximum output). ${ }^{9}$ While some vibrating mesh nebulizers can apparently function reliably above $12 \mathrm{~mL} / \mathrm{h}$, knowing which devices can do this would require clinicians to pretest vibrating mesh nebulizers before use.

The distribution of data points in Figure 2 shows that the scatter of the data, as a function of the 4 different ventilator duty cycles, appears to be minimal at lower infusion pump flows and increases as the infusion pump flow increases. This was true for both nebulizer types. This variability may be due to effects of duty cycle on nebulizer output. For continuous nebulization, it is known that aerosol delivery is enhanced as duty cycle is increased. ${ }^{11}$ This effect, while significant, was small (Table 3).

Observations of vibrating mesh nebulizer failures during aerosol studies, off and on mechanical ventilation, have been previously reported. ${ }^{11,14}$ In the study by Gowda et al, ${ }^{14}$ a sporadic failure rate of $25 \%$ was noted during a pre-protocol observational exercise and $30 \%$ during the experimental protocol with $3 \mathrm{~mL}$ normal saline fill volumes. The failures were attributed to random cessation of nebulization, resulting in failure of the device to empty. Although the manufacturer specifies a residual volume of $<0.1 \mathrm{~mL}$ (3.3\% of nebulizer charge volume), ${ }^{8}$ the study by Gowda et al accepted a residual volume of $0.3 \mathrm{~mL}$ (ie, $10 \%$ of nebulizer charge volume). ${ }^{14}$ In a recent study by our group, which compared 3-and 6-mL fill volumes for aerosol therapy during mechanical ventilation with breath-enhanced jet nebulizers and vibrating mesh nebulizers, the vibrating mesh nebulizer failed to empty in $55 \%$ of the test runs. ${ }^{11}$

The mass balance data (Table 4) indicate that connecting a nebulizer to the dry side of the humidifier can result in significant amounts of drug deposition in the humidifier and confirms that wet-side nebulizer mounting does not significantly contaminate the humidifier. Humidifier deposition was measured at $15.3 \%$ of nebulizer delivery for the vibrating mesh nebulizer on the dry side versus $0.2 \%$ of nebulizer delivery for the breath-enhanced jet nebulizer on the wet side. Ashraf et $\mathrm{al}^{11}$ made similar observations for $3-\mathrm{mL}$ nebulizer fill volumes during mechanical ventilation: humidifier deposition for vibrating mesh nebulizer (dry side) was $17.1 \%$ of nebulizer charge and $0 \%$ of nebulizer charge for the breath-enhanced jet nebulizer on the wet side.

Aerosol particle size distributions were similar for both technologies, with mean MMAD at the distal tip of the endotracheal tube of $2.54 \mu \mathrm{m}$ for the vibrating mesh nebulizer and $2.52 \mu \mathrm{m}$ for the breath-enhanced jet nebulizer. While these distributions are in the respirable range, the breathenhanced jet nebulizer data during continuous infusion permitted larger particles than reported by our group for other forms of mechanical ventilation. Cuccia et $\mathrm{al}^{10}$ reported MMAD for a ventilator-breath-actuated/breath-enhanced jet nebulizer to be $1.34 \mu \mathrm{m}$ with humidification. In a study of continuous nebulization with a conventional fill volume $(3 \mathrm{~mL})$ for the breath-enhanced jet nebulizer, Ashraf et $\mathrm{al}^{11}$ measured an MMAD of $1.95 \mu \mathrm{m}$, also with humidification. Similarly, Ashraf et al ${ }^{11}$ also reported an MMAD of 1.90 $\mu \mathrm{m}$ for the vibrating mesh nebulizer with humidification (dry-side mounting). These data suggest that aerosol delivery by continuous infusion produces somewhat larger particle distributions for both nebulizer technologies.

Previous papers have described the clinical use of various jet nebulizers for continuous infusion aerosol delivery, but they did not measure delivered aerosol. ${ }^{1-6}$ In 2003, Siobal and colleagues ${ }^{7}$ conducted a bench study of a novel method of delivering aerosolized prostacyclin using 2 infusion pumps connected together to infuse a normal saline and epoprostenol mixture into a MiniHEART Lo-Flo nebulizer. Delivered epoprostenol dosage was titrated by changing the ratio of the infusion flows of normal saline to epoprostenol solution while maintaining a total infusion pump flow of $8 \mathrm{~mL} / \mathrm{h}$ to match the nebulizer output rate. Using albuterol as a surrogate for epoprostenol, they collected aerosol on a filter at the distal tip of the endotracheal tube and determined an average inhaled mass for 5 MiniHEART Lo-Flo nebulizers of $14 \%$ of the nominal dose placed in the nebulizer. However, the significance of this finding is uncertain inasmuch as the assessment was performed over test runs of only 5 min extrapolated to $1 \mathrm{~h}$, and the tests were not conducted during actual infusion of solution into the nebulizer and therefore is not directly comparable to the present study.

A recent bench study by Anderson et $\mathrm{al}^{9}$ represents the only in vitro assessment of vibrating mesh technology for continuous infusion aerosol delivery in the literature. Using a single vibrating mesh nebulizer, selected from a group of pre-tested nebulizers and confirmed to have an output of $20.2 \mathrm{~mL} / \mathrm{h}$, Anderson and colleagues sought to distinguish specific differences in aerosol delivery at 4 positions in the ventilator circuit. ${ }^{9}$ They used the vibrating mesh nebulizer to nebulize epoprostenol at 3 different delivery rates (ie, 30, 50, and $70 \mathrm{ng} / \mathrm{kg} / \mathrm{min}$ ), which corresponded to infusion pump flows of 8.4, 14.0, and $19.6 \mathrm{~mL} / \mathrm{h}$, respectively, for a $50-\mathrm{kg}$ patient model. Based upon the infusion syringe concentration and infusion pump flows, they calculated what they termed as the expected total dose (ng) of epoprostenol aerosol that would be collected on a filter at the distal tip of the endotracheal tube in $20 \mathrm{~min}$ for both dry-side and wet-side humidifier positioning of a vibrating mesh nebulizer, as well as 2 other positions. Subsequently, the measured epoprostenol aerosol mass delivered to the filter, reported as the mean total dose and expressed as a percentage of the expected total dose, ranged from $15.1 \%$ to $19.1 \%$ for dryside humidifier mounting and from $15.6 \%$ to $17.7 \%$ for wet-side humidifier mounting across the 3 infusion pump 


\section{Continuous Infusion Aerosol Delivery}

flows studied. What Anderson et $\mathrm{al}^{9}$ reported as mean total dose (ng/20 min) can also be expressed, as we have done in this study, as inhaled mass because it represented the mass of epoprostenol delivered to the collection filter in their model. When we graphed their inhaled mass (ie, mean total dose) against infusion pump flow, there was a positive slope and linear relationship. They reported that the differences in mean total dose between the dry-side position and the wet-side position were not statistically significant.

Similarly, the quotient of mean total dose divided by expected total dose, which they reported as \% delivered, could also be expressed as inhaled mass \% (as a percentage of the nebulizer charge) if it is assumed that the mass of epoprostenol delivered to the nebulizer for aerosolization represents the nebulizer charge. Consequently, their inhaled mass $\%$ ranged from $15.1 \%$ to $19.1 \%$ (mean $=16.7 \%)$ for the 3 dosing scenarios reported for both dry-side and wetside experiments, which is roughly half of what was determined for inhaled mass in our mass balance experiment for the vibrating mesh nebulizer (31.8\%, shown in Table 3 ).

There are 3 possibly interacting reasons for this difference. First, ventilator settings and breathing patterns are not comparable between the 2 studies. Our experiments used 4 different breathing patterns with duty cycles (\% inspiratory time) ranging between 0.13 and 0.34 (Table 1). Anderson et $\mathrm{al}^{9}$ used 3 different breathing patterns, all with essentially the same, relatively short, duty cycle (calculated from their ventilator settings) ranging between 0.134 and 0.142 . In a previous study by our group, we reported a positive trend between duty cycle and inhaled mass. ${ }^{10}$ The higher duty cycles in many of our experiments may be a contributing factor to higher inhaled mass for the breath-enhanced jet nebulizer. Second, Anderson et $\mathrm{al}^{9}$ used a ventilator bias flow of $4.5 \mathrm{~L} / \mathrm{min}$, more than twice the $2.0-\mathrm{L} / \mathrm{min}$ bias flow used in our experiments. Our laboratory ${ }^{16}$ and other investigators ${ }^{17}$ have reported that inhaled mass is reduced with higher bias flows. Therefore, their higher bias flow may also have contributed to the decreased inhaled mass for the vibrating mesh nebulizer. Third, there could be higher humidifier or tubing losses, although this cannot be confirmed from the study by Anderson et $\mathrm{al}^{9}$ study because they did not perform a mass balance measurement.

The study by Anderson et $\mathrm{al}^{9}$ study also reported nearly identical mean total dose values for epoprostenol collected on the filter: $6,347 \pm 973 \mathrm{ng}$ for the dry side versus $6,537 \pm 2,234 \mathrm{ng}$ for the wet side of the humidifier. Based on our mass balance data, which showed approximately $15 \%$ deposition in the humidifier due to dry-side mounting, it could be expected that the inhaled mass for the vibrating mesh nebulizer would be higher with wet-side mounting because no humidifier deposition would have occurred. However, it is likely that there was greater deposition downstream of the humidifier, thereby rendering roughly the same inhaled mass for both wet-side and dry-side mounting of the vibrating mesh nebulizer. The latter phenomenon has been reported by our group when comparing circuit deposition with and without active humidification. ${ }^{11}$

\section{Limitations}

An important limitation of this study is that it is a bench study rather than a clinical study. A second limitation is that we nebulized normal saline rather than an available epoprostenol product such as Flolan (GlaxoSmithKline, Research Triangle Park, North Carolina) or Veletri (Actelon, San Francisco, California). Clinicians have reported that the glycine diluent in Flolan makes some ventilator exhalation valves sticky, causing them to malfunction, while Veletri does not have that issue. ${ }^{9}$ However, both drugs are aqueous solutions with physicochemical behavior in the nebulizer similar to normal saline. ${ }^{13}$

\section{Conclusions}

In vitro study of continuous infusion aerosol delivery during adult mechanical ventilation and multiple regression analysis of the variables indicate that inhaled mass is primarily influenced by infusion pump flow and, to a much lesser degree, by ventilator duty cycle, but not nebulizer type. Aerosol delivery comparable to a properly functioning vibrating mesh nebulizer can be achieved with a breath-enhanced jet nebulizer, and the InspiRx iAIRE breath-enhanced jet nebulizer exhibited greater reliability than the Aerogen Solo vibrating mesh nebulizer. Our results suggest that the breath-enhanced jet nebulizer can be used interchangeably with existing continuous infusion vibrating mesh nebulizer protocols.

\section{ACKNOWLEDGMENTS}

The authors acknowledge the artistic, statistical, and technical assistance of Lorraine Morra in the preparation of this paper and the assistance of the Respiratory Care Department at University Medical Center, Stony Brook, for the loan of equipment and supplies.

\section{REFERENCES}

1. Walmrath D, Schneider T, Pilch J, Grimminger F, Seeger W. Aerosolized prostacyclin in adult respiratory distress syndrome. Lancet 1993;342(8877):961-962.

2. Zwissler B, Kemming G, Habler O, Kleen M, Merkel M, Haller M, et al. Inhaled prostacyclin (PG12) versus inhaled nitric oxide in adult respiratory distress syndrome. Am J Respir Crit Care Med 1996;154(6 Pt 1):1671-1677.

3. Meyer J, Theilmeier G, Van Aken H, Bone HG, Busse H, Waurick R, et al. Inhaled prostaglandin $\mathrm{E}_{1}$ for treatment of acute lung injury in severe multiple organ failure. Anesth Analg 1998;86(4):753-758. 


\section{Continuous Infusion Aerosol Delivery}

4. Van Heerden PV, Barden A, Michalopoulos N, Bulsara MK, Roberts $\mathrm{BL}$. Dose-response to inhaled aerosolized prostacyclin for hypoxemia due to ARDS. Chest 2000;117(3):819-827.

5. Haché M, Denault AY, Bélisle S, Couture P, Babin D, Tétrault F, Guimond JG. Inhaled prostacyclin $\left(\mathrm{PGI}_{2}\right)$ is an effective addition to the treatment of pulmonary hypertension and hypoxia in the operating room and intensive care unit. Can J Anaesth 2001;48(9):924-929.

6. Haché M, Denault A, Bélisle S, Robitaille D, Couture P, Sheridan P, et al. Inhaled epoprostenol (prostacyclin) and pulmonary hypertension before cardiac surgery. J Thorac Cardiovasc Surg 2003;125(3):642-649.

7. Siobal MS, Kallet RH, Pittet J-F, Warnecke EL, Kraemer RW, Venkayya RV, Tang JF. Description and evaluation of a delivery system for aerosolized prostacyclin. Respir Care 2003;48(8):742-753.

8. Aerogen. Aerogen Solo System Instruction Manual, P/N AG-AS3050US, P/N 30-674 Rev H. Galway, Ireland: Aerogen; 2017.

9. Anderson AC, Dubosky MN, Fiorino KA, Quintana V, Kaplan CA, Vines DL. The effect of nebulizer position on aerosolized epoprostenol delivery in an adult lung model. Respir Care 2017;62(11):1387-1395.

10. Cuccia AD, Ashraf S, McPeck M, Samuel J, Smaldone GC. Wet side breath-enhanced jet nebulization: controlling drug delivery during mechanical ventilation. Respir Care 2020;65(8):1077-1089.
11. Ashraf S, McPeck M, Cuccia AD, Smaldone GC. Comparison of vibrating mesh, jet, and breath-enhanced nebulizers during mechanical ventilation. Respir Care 2020;65(10):1419-1426.

12. McPeck M, Tandon R, Hughes K, Smaldone GC. Aerosol delivery during continuous nebulization. Chest 1997;111(5):1200-1205.

13. Harris KW, O'Riordan TG, Smaldone GC. Aerosolized iloprost customized for the critically ill. Respir Care 2007;52(11):15071509.

14. Gowda AA, Cuccia AD, Smaldone GC. Reliability of vibrating mesh technology. Respir Care 2017;62(1):65-69.

15. Sagalla R, Smaldone GC. Capturing the efficiency of vibrating mesh nebulizers: minimizing upper airway deposition. J Aerosol Med Pulm Drug Deliv 2014;27(5):341-348.

16. Miller DD, Amin MM, Palmer LB, Shah AR, Smaldone GC. Aerosol delivery and modern mechanical ventilation: in vitro/in vivo evaluation. Am J Respir Crit Care Med 2003;168(10):1205-1209.

17. Ari A, Atalay OT, Harwood R, Sheard MM, Aljamhan EA, Fink JB. Influence of nebulizer type, position, and bias flow on aerosol drug delivery in simulated pediatric and adult lung models during mechanical ventilation. Respir Care 2010;55(7):845-851.

This article is approved for Continuing Respiratory Care Education credit. For information and to obtain your CRCE

(free to AARC members) visit 